\title{
Understanding an Ontology through Divergent Exploration
}

\author{
Kouji Kozaki, Takeru Hirota, and Riichiro Mizoguchi \\ The Institute of Scientific and Industrial Research, Osaka University \\ 8-1 Mihogaoka, Ibaraki, Osaka, 567-0047 Japan \\ \{kozaki, hirota,miz\}@ei.sanken.osaka-u.ac.jp
}

\begin{abstract}
It is important that the ontology captures the essential conceptual structure of the target world as generally as possible. However, such ontologies are sometimes regarded as weak and shallow by domain experts because they often want to understand the target world from the domain-specific viewpoints in which they are interested. Therefore, it is highly desirable to have not only knowledge structuring from the general perspective but also from the domainspecific and multi-perspective so that concepts are structured for appropriate understanding from the multiple experts. On the basis of this observation, the authors propose a novel approach, called divergent exploration of an ontology, to bridge the gap between ontologies and domain experts. Based on the approach, we developed an ontology exploration tool and evaluated the system through an experimental use by experts in an environmental domain. As a result, we confirmed that the tool supports experts to obtain meaningful knowledge for them through the divergent exploration and it contributes to integrated understanding of the ontology and its target domain.
\end{abstract}

Keywords: ontology, divergent exploration, view point, conceptual map.

\section{Introduction}

Ontologies are designed to provide underlying conceptual structure and machine readable vocabulary of domains for Semantic Web applications. Ontology is defined as "An explicit specification of conceptualization"[1], and it clearly represents how the target world is captured by people and systems. That is, an ontology construction implies to understand the target world, and understanding the ontology means understanding the target world to some extent. Especially, an ontology plays an important role for comprehensive understanding of a complex domain which consists of many sub-domains. To use an ontology for this purpose, there are two approaches: 1) mapping ontologies which are built for each domain, and 2) building domain ontlogies based on the same shared upper ontology. While the former is easily acceptable to domain experts, ontology mapping is a hard task and needs large cost. To avoid the mapping issue, many research groups take the latter approach. OBO Foundry coordinates activities to develop and share ontologies which cover common concepts across domains[2]. That is, knowledge sharing and exchanging across domains can be realized through the shared common vocabulary defined in the same ontology. 
For knowledge sharing through an ontology, it is important that the ontology captures the essential conceptual structure of the target world as generally as possible and they should be well organized with consistency and reusability. Even if it is domain-dependent and specialized concept, its meaning could be shared with people working in other domains through its definition in terms of generalized common concepts. For example, when we define a specialized relationship well-known in a domain, it could be represented as combinations of general relationships by decomposing the original relation. The generalized representation makes implicit knowledge explicit and machine readable one. In this way, an ontology contributes to readability and interoperability of knowledge through domain-independent and generalized conceptualization. However, such generalized ontologies are sometimes regarded as verbose and shallow by domain experts because they often want to understand the target world from the domain-specific viewpoints in which they are interested. In many cases their interests are different and versatile, even if they are experts in the same domain. It is a serious and important issue to bridge the gap between ontologies which try to cover wide area domain-independently and interests of domain experts which are well-focused and deep. Therefore, it is highly desirable to have not only knowledge structuring from the general perspective but also from the domain-specific and multi-perspective so that concepts are structured for appropriate understanding from the multiple experts.

On the basis of this observation, the authors propose a novel approach to bridge the gap between ontologies and domain experts. The main strategy is composed of: (1) the conceptual structure of an ontology is systematized as generally as possible and (2) on the fly reorganizing some conceptual structures from the ontology as visualizations to cope with various viewpoints which reflects interests of the domain experts (Fig.1). Based on this strategy, we developed a frame work, named divergent exploration of ontology, and an ontology exploration tool as implementation of it. The tool allows users to explore an ontology according to their own perspectives and visualizes them in a user-friendly form, i.e. conceptual map. It contributes to helping users explore the ontology from several viewpoints to eventually obtain integrated understanding of the ontology and its target domain. Furthermore, it stimulates their intellectual interest and could support idea creation.

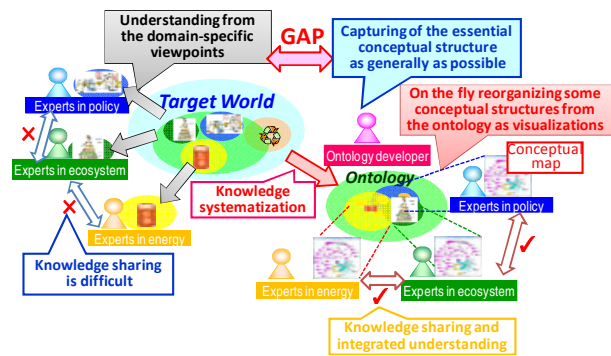

Fig. 1. Integrated understanding of crossdomain knowledge through divergent exploration of an ontology

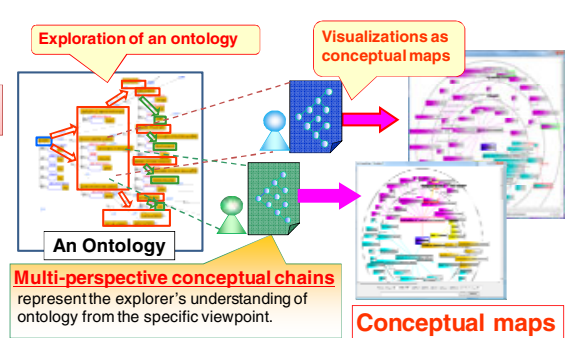

Fig. 2. Divergent exploration of ontology 
The rest of this paper is organized as follows: In section 2, we introduce divergent exploration for understanding an ontology from multi-perspectives. After we discuss requirements for the ontology exploration tool, we discuss its functionality in section 3. In Section 4, we evaluate the system through its application to an environmental domain and an experimental use by domain experts. In section 5, we summarize some related works. Finally, we present concluding remarks with future work.

\section{Divergent Exploration}

\subsection{Divergent Exploration of an Ontology}

Most of semantic web applications use ontologies as vocabularies to describe metadata and are aimed at semantic processing of them. By contrast, we regard ontology as the target for divergent exploration of the ontology itself. The divergent exploration of an ontology enables users to explore a sea of concepts in the ontology freely from a variety of perspectives according to their own motive. The exploration stimulates their way of thinking and contributes to deeper understanding of the ontology and hence its target world. As a result, the users can find out what they take interest. Some of them could include new findings for them because they could obtain unexpected conceptual chains which they have never thought through the ontology exploration.

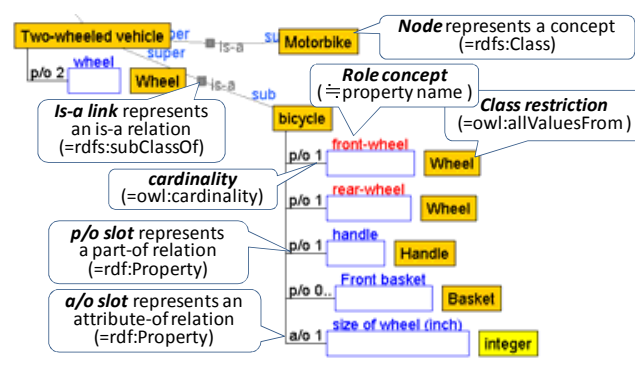

Fig. 3. An example ontology representation in Hozo and its correspondence with OWL
Table 1. The aspects of ontology exploration for each relationship

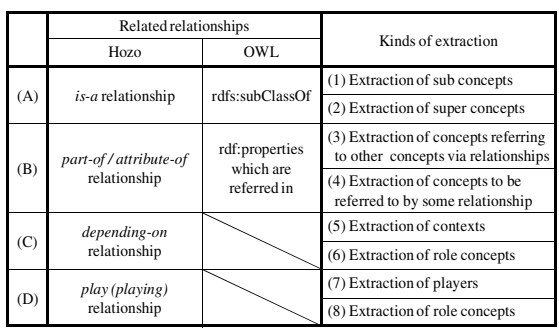

Fig.2 outlines the framework of ontology exploration. The divergent exploration of an ontology can be performed by choosing arbitrary concepts from which, according to the explorer's intention, they trace what we call multi-perspective conceptual chains. We define the viewpoint for exploring an ontology and obtaining the multi-perspective conceptual chains as the combination of a focal point and aspects. The focal point indicates a concept to which the user pays attention as a starting point of the exploration. The aspect is the manner in which the user explores the ontology. Because an ontology consists of concepts and the relationships among them, the aspect can be represented by a set of methods for extracting concepts according to its relationships. The multi-perspective conceptual chains are visualized in a user-friendly form, i.e., in a conceptual map. Although there are many researches 


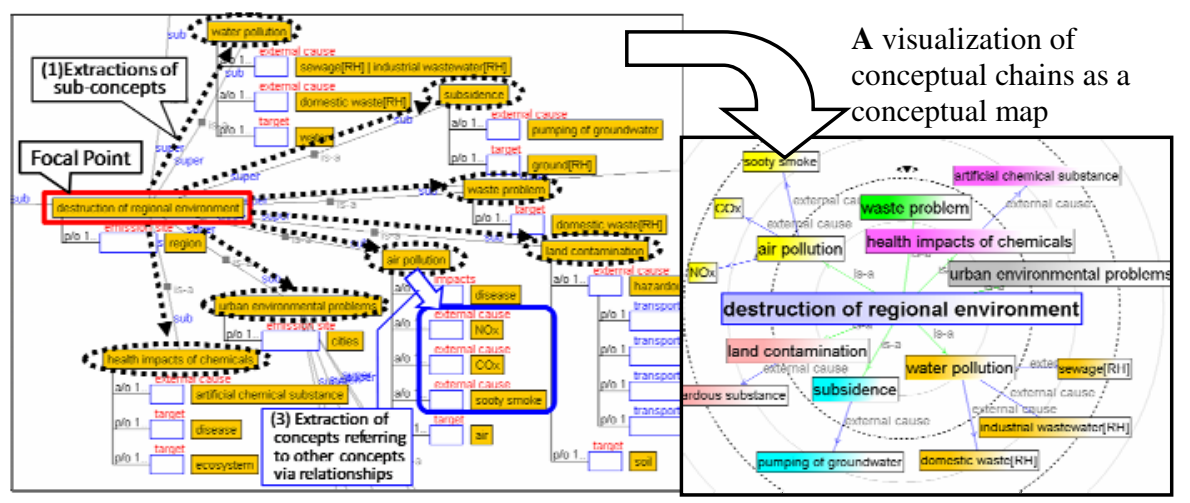

Fig. 4. An example of divergent exploration of an ontology

on visualization of ontology, the main purpose of our research is not the visualization itself but exploration of an ontology. It is neither ontology browsing which are supported by most of ontology development tool nor ontology summarization. The divergent exploration of an ontology aims at integrated understanding of the ontology and its target world from multiple perspectives across domains according to the users' interests. We also focus on that our tool supports domain experts to obtain meaningful knowledge for themselves as conceptual chains through the divergent exploration.

\subsection{Definition of View Points}

We implement the divergent exploration of an ontology as an additional function of Hozo which is our ontology development tool[3]. Fig.3 shows an example of ontology defined using Hozo. Ontologies are represented by nodes, slots and links. The nodes represent concepts (classes), is-a links represent is- $a$ (subclass-of) relations, and slots represents part-of (denoted by "p/o") or attribute-of (denoted by "a/o") relations. A slot consists of its kind ("p/o" or "a/o"), role concept, class restriction, cardinality. Roughly speaking, a slot corresponds to property in OWL and its role name represent name of property. Its class restriction and cardinality correspond owl:allValuesFrom and owl:cardinality respectively. However, semantics of Hozo's ontology includes some concepts related in role which are not supported in OWL because it is designed based on ontological theory of role[4]. While we have designed three levels of role representation model in OWL to capture the semantics level-wise [5], we use simplest model described above in this paper.

As described above, viewpoints are defined as a combination of a focal point and aspects, and an aspect is represented according to relationships defined in an ontology. We classify these relationships into four kinds and define two aspects of ontology exploration for each relationship according to the direction to follow (upward or downward) (See Table.1). The user can control kinds of relationships to follow by specifying kinds of role concept (properties) in the aspects type (B) to (D). We call the control "role limitation of aspect". Similarly, users can constrain the types 
of concepts to reach through aspects by specifying types of concepts. We call the constraint "class type limitation of aspect". While we can suppose more detailed viewpoints such as limitation of cardinality, we do not introduce them because they are too be detailed for domain experts to explore easily.

Fig.4 shows an example of an ontology exploration. The user set Destruction of regional environment as the focal point and select (1) Extraction of sub concepts as an aspect. Then, following is-a relations, seven concepts such as Air pollution, Land contamination, etc. are extracted. Next, if the user focus on Air pollution and selects (3) Extraction of concepts referring to other concepts via relationships as an aspect, Disease, NOx, COx, Sooty smoke and Air are extracted following attribute-of relations of Air pollution. On the other hand, if the user applies external cause as role limitation of aspects, only NOx, COx and Sooty smoke, which are related to external cause, are extracted (Fig. 4 left). As a result of this concept extraction, the system generates conceptual chains that match the user's interest and visualizes them as a conceptual map. In the conceptual map, extracted concepts and followed relationships are represented as nodes and links respectively, and the nodes are located on concentric circles in which the focal point is located at the center. As a result, the conceptual chains are represented as a divergent network (Fig.4 right). In this way, the user can explore an ontology from various viewpoints by choosing combinations of focal points and aspects, and the results are visualized as conceptual maps.

\subsection{Requirements for the Ontology Exploration Tool}

In this section, we discuss functions which the ontology exploration tool is required to support. The viewpoints for ontology exploration are defined by combination of focal points and aspects. In many cases, the combination is fixed through repetitions of choice of aspects for the exploration by trial and error because not every user has clear intentions and viewpoints to explore the ontology at first. That is, the user explores the ontology step by step and clarifies his/her interest gradually. The result of this process is represented as multi-perspective conceptual chains. Functions to support such a step by step exploration of the user are required as the most fundamental function of the ontology exploration tool.

On the other hands, it is required to make full use of semantic processing, which is a feature of ontology, for exploration. For example, a function to search all combinations of aspects automatically and get conceptual chains which represents paths from a focused concept to another specified concept, we call it search path function, seems to be useful. Functions to investigate obtained conceptual maps from additional perspectives such as change view and comparison function are also required. The change view is a function to apply additional viewpoints to a conceptual map generated based on another viewpoint, and as a result the visualization of the map is changed according to the specified concepts or relationships in the additional viewpoint. And a comparison function enables the user to compare two conceptual maps and visualize the common elements in them. 


\section{Implementation of the Ontology Exploration Tool}

\subsection{System Architecture}

Fig.5 shows the architecture of the ontology exploration tool. It consists of aspect dialog, concept extraction module and conceptual map visualizer. The aspect dialog provides graphical user interface to select viewpoints for ontology extraction. The concept extraction module follows relationships between concepts according to the selected viewpoint and obtains multi-perspective conceptual chains. The conceptual chains are visualized as conceptual maps by conceptual map visualizer. While the target of the system

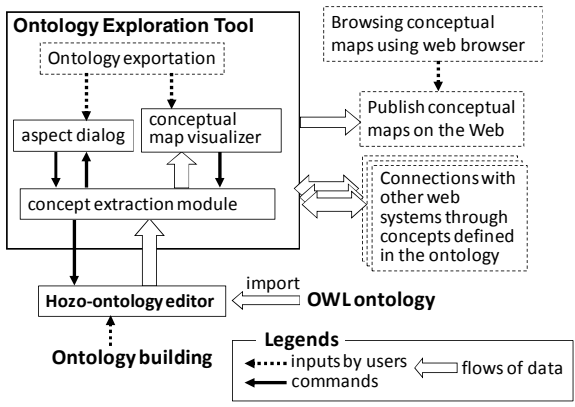

Fig. 5. The architecture of ontology exploration tool is an ontology in Hozo's format, it also can support an ontology in OWL because Hozo can import OWL ontology. The generated conceptual maps can be connected with other web systems through concepts defined in the ontology. For example, mapping nodes in the conceptual map with Linked Data allows the user to access various web resources through it. While the ontology exploration tool is implemented as a client application by Java, it can export generated maps in an XML format and publish them on the Web. Users can browse them using web browsers with a conceptual map viewer implemented by Flash. Demos of browsing the conceptual map and download of the system are available at the URL: http://www.hozo.jp/OntoExplorer/ .

\subsection{Functions for Ontology Exploration}

The ontology exploration tool provides the following functions (Fig.6).

Detailed exploration using aspect dialog: The aspect dialog lists kinds of aspect with numbers of concept which will be extracted when the aspect is selected. The user selects an aspect with detailed setting for exploration such as role limitation and extracting class type limitation. The user can explore from detailed viewpoint by repetition of selecting aspects with the settings.

Simple exploration: The conceptual map visualizer provides commands to apply some typical combinations of aspects as mouse menus. The user can explore an ontology by simple operation using them. While this operation does not allow detailed settings, we suppose beginners of the tool can use it and explore easily.

Search path (machine exploration): The system can search all combination of aspects to generate conceptual chains from a concept selected as starting point to those specified by the user. As a result, the system shows all conceptual chains between the selected concepts.

Change view: The tool has a function to highlight specified paths of conceptual chains on the generated map according to given viewpoints. For example, when the user specifies a focusing concept, the conceptual map visualizer highlights the paths 
which include the concepts or its sub concepts in them. It also can display only the highlighted paths on the map by hiding other paths. Another viewpoint for highlighting includes specifying the focusing kinds of relationships (aspects) and specifying concepts or relationships which has some values of attributes. When the user specifies several viewpoints at the same time, the system highlights paths in different colors according to the viewpoints. Through this function, the user can switch several viewpoints easily and compare difference between them. For example, when we suppose a conceptual map which represents effects of global warming, the user can change viewpoints according to time scale which the effects will occur and/or spatial extent of them.

Comparison of maps: The system can compare generated maps and show the common conceptual chains both of the maps.

\section{Usage and Evaluation of Ontology Exploration Tool}

\subsection{Usage for Knowledge Structuring in Sustainability Science}

Sustainability science (SS) is a discipline aimed at establishing new disciplinary schemes that serve as a basis for constructing a vision that will lead global society to a sustainable one. Meeting this objective requires an interdisciplinary integrated

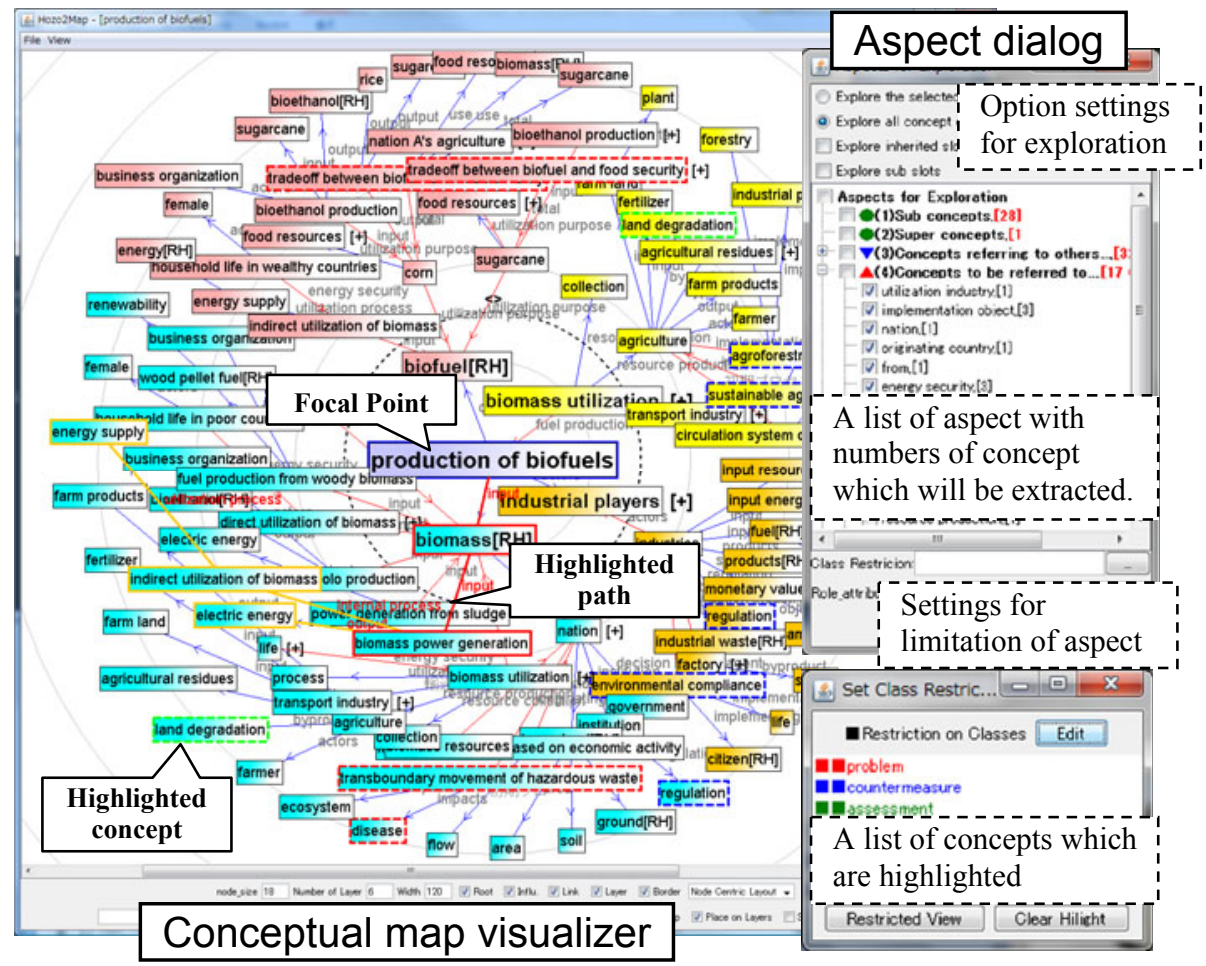

Fig. 6. A snapshot of the ontology exploration tool 
understanding of the entire field instead of knowledge structuring that depends on individual related domains. Thus, Osaka University Research Institute for Sustainability Science (RISS) has been working on construction of an SS ontology in which knowledge of domains relating to SS is organized into a common domainindependent conceptual structure. Furthermore, with the ontology exploration tool prototype developed in this research, by generating conceptual maps from the SS ontology in accordance with users' viewpoints, attempts were at knowledge structuring in sustainability science, such as knowledge sharing among experts in different domains and an integrated understanding from multiple viewpoints. As a result, through usage of the tool by experts at RISS, it was confirmed that the tool had a certain utility for achieving knowledge structuring in sustainability science [6]. The ontology exploration tool described in Section 3 is a tool for enabling an overview of multiple domains, improved on the basis of the above findings. Furthermore, in this research, we conducted evaluation in a setting closer to that used in practice.

\subsection{Verification of Exploring Ability of Ontology Exploration Tool}

In order to verify whether the tool can properly explore an ontology and generate conceptual maps that domain experts wish to do, we enriched the SS ontology in a more specific domain (biofuels) and verified the exploring ability of the tool.

\subsubsection{Enrichment of SS Ontology}

First, we constructed a Biofuel ontology by enriching the SS ontology described in Section 4.1 with concepts relating to biofuels. Before enriching the ontology, from base material collected by reviewing existing research, domain experts organized the structures of target problems in ontology construction into 44 typical scenarios representing instances of how the production and usage of biofuels affect various fields. Each of the scenarios was
Table 2. The numbers of scenarios

\begin{tabular}{|l|c|}
\hline \multicolumn{1}{|c|}{ Problem category } & $\begin{array}{c}\text { Number of } \\
\text { scenarios }\end{array}$ \\
\hline \hline 1) Energy services for the poor & 3 \\
\hline $\begin{array}{l}\text { 2) Agriculture and industry development and } \\
\text { employment creation }\end{array}$ & 6 \\
\hline 3) Health and gender & 4 \\
\hline 4) Agricultural structures & 4 \\
\hline 5) Food security & 6 \\
\hline 6) Government budget & 4 \\
\hline 7) Trade, foreign exchange balance, energy security & 5 \\
\hline 8) Biodiversity and natural resource management & 8 \\
\hline
\end{tabular}
expressed in the form of a short sentence, such as "(1-3) Biofuels can replace only a small share of the global energy supply, and biofuels alone are not sufficient to overcome our dependence on fossil fuels" or "(2-6) Small-scale labor-intensive bioenergy production is effective in creating employment but has drawbacks with production efficiency and economic competitiveness (tradeoff relationship)." The scenarios are classified into the nine categories listed in Table 2.

Then, on the basis of the contents of these scenarios, keeping in mind that the enriched ontology should serve for conceptual map generation, ontology experts took the following procedure:

1) Add main concepts appearing in the scenarios to the ontology.

2) Clarify relationships among the concepts appearing in the contents of the scenarios, including between-the-lines relationships (hidden causal chains) not explicit in the text of the scenarios.

3) Describe the relationships clarified in step 2 as an ontology. 
For example, in the case of the scenario "(4-1) Demand for energy crop production increases pressure on land (farms) for food production, resulting in a rise in food prices," concepts added to the ontology in step 1 were Demand for biomass resources, Farms for food production, and Rise in food prices, and the relationship clarified in step 2 was "Increase in demand for biomass resources $\rightarrow$ Increase in farms for fuel production $\rightarrow$ Problem of fixed area $\rightarrow$ Decrease in farms for food production $\rightarrow$ Decrease in food supply $\rightarrow$ Rise in food prices." When we added concepts and relationships in ontology, we defined not only them but also upper concepts of them. For example, relationships between farms for fuel production and farms for food production were generalized as a finite total amount. These generalized definitions enable the users to generate a wide variety of conceptual maps which are not directly represented in the scenario.

Through such examination, we reorganized the 44 scenarios listed in Table 1 into 29 scenarios, in consideration of complex relationships and similarity of relationships, and enriched the ontology for the 29 scenarios. The scale of the resulting biofuel ontology was about twice as large as the scale of the SS ontology described in Section 4.1; specifically, the number of concepts increased from 649 to 1892, and the number of slots increased from 1075 to 2119 .

\subsubsection{Verification of Scenario Reproducing Operation}

We verified whether the ontology enriched by the method described above can properly express the original problem structures by examining whether it was possible, with the ontology exploration tool, to generate conceptual maps in which the contents of the original scenarios were reproduced. As exploration (viewpoint setting) methods used for map generation, the following three methods were attempted. As a result, the number of original scenarios for which conceptual maps corresponding to the problem structures represented by the scenarios were successfully generated (reproduced) and the ratio of such scenarios to the total number of scenarios were as follows:

1) Scenarios reproduced by exploration with Search Path function (automatic exploration of combinations of relationships among all the concepts): 21 (72\%)

2) Scenarios reproduced by simple exploration (exploration is performed by using only simplified exploration conditions (aspects), and the Change View function for extracting only the paths including specified concepts is used): 24 (82\%)

3) Scenarios reproduced by simple exploration or detailed exploration (exploration performed with a selected detailed viewpoint): 27 (93\%)

As for the two scenarios that were not reproduced, we found that inadequate ontology definition was the reason for the failure to reproduce conceptual maps corresponding to these scenarios. That is, the two scenarios could not reproduced as conceptual maps not because ability of the tool but because we missed to add some relationships in the two scenarios. In short, we can conclude that the exploration ability and conceptual map expressing ability of the tool were sufficient for reproducing target scenarios in the form of conceptual maps. 


\subsection{Experiment for Evaluating Ontology Exploration Tool}

\subsubsection{Experiment Overview}

In order to verify that the ontology exploration tool developed in this research is useful for obtaining a domain overview, we conducted an evaluation experiment with cooperation from experts. In the experiment, we used the Biofuel ontology described in Section 4.3. The experiment was aimed at evaluating the following two issues through actual usage of the tool by the experts:

1) Whether meaningful maps that provide intellectual stimulation were obtained.

2) Whether meaningful maps other than those representing the contents of the scenarios anticipated at the time of ontology construction were obtained.

The subjects of the experiments were four experts $A$ to $D$ in different fields of expertise. The experts A and B had no experience of using the tool at all, and the experts $\mathrm{C}$ and $\mathrm{D}$ had some experience. The fields of expertise of the individual experts were as follows; A: Agricultural economics, B: Social science (stakeholder analysis), C: Risk analysis, D: Metropolitan environmental planning. Although someone may suppose just four persons are too few for evaluation, note that they are neither students nor public users which can be easily collected in usual cases. We believe that the experiments by four real experts are meaningful as an initial evaluation of the tool.

\subsubsection{Experimental Method}

First, we asked the four experts to generate conceptual maps with the tool in accordance with condition settings of the following tasks 1 to 3 .

Task 1: Under the supposed problem "What kinds of Environmental destruction problems relating to Biofuels exist," conceptual maps were generated under the conditions that the concept that could be selected first (Focal Point) was restricted to Bioenergy usage or Biomass resource production and the exploration method was either 1) automatic exploration by Search Path function or 2) simple exploration described in Section 4.2.2. At this time, the experts were allowed to freely select concepts used for viewpoint setting with Search Path or Change View from sub concepts of Environmental destruction problems.

Task 2: "What kinds of Tradeoff problems relating to Biofuels exit?" We asked the experts to generate conceptual maps under the same conditions as those in Task 1, except that they were allowed to freely select concepts used for viewpoint setting with Search Path or Change View from sub concepts of Tradeoff problems.

Task 3: We asked the experts to generate conceptual maps by arbitrary methods regarding problems related to their interests.

Then, from the paths of conceptual chains (visualized paths that track a series of relationships among concepts) included in the conceptual maps generated, paths that were clearly judged as inappropriate by the experts were removed, and evaluation was conducted using the paths selected by the subjects according to their interests. Note here, although we restricted operations for exploration in Task 1 and 2, the number of 
combinations allowed for the subjects were 184 and 64 respectively at least ${ }^{l}$. Furthermore, they did not know the contents of scenarios which we supposed in each task. Therefore, the selected paths did not always correspond to the scenarios. Then we asked the subjects to enter, via a special input screen on the tool, a four-level general evaluation (A: Interesting, B: Ordinary but important, C: Neither good or poor, D: Obviously wrong), a four-level evaluation (Excellent, Good, Normal, Bad) regarding four specific points (1: Clarity, 2: Faithful reproduction, validity, and appropriateness, 3: Ease of overview, coverage, and comprehensiveness, 4: Conception and discovery assistance (stimulation)), and free comments.

Table 3. Experimental results

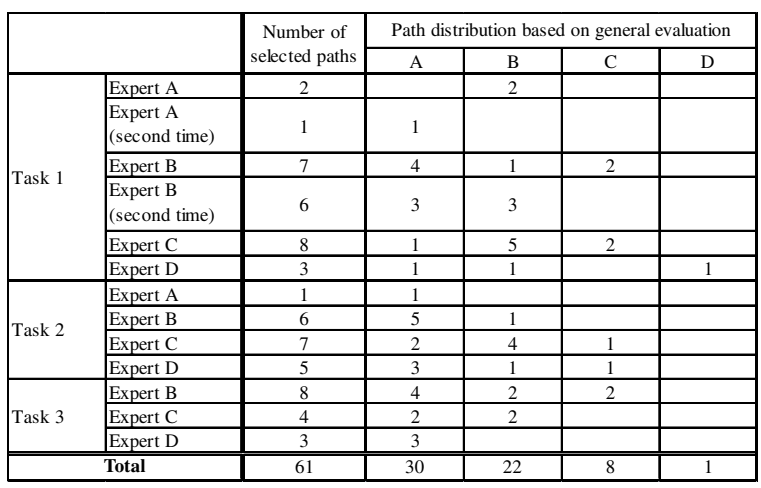

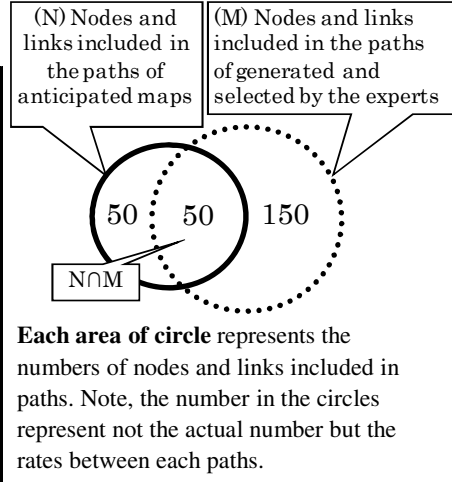

Fig. 7. The rate of paths

\subsubsection{Experimental Results and Discussion}

As results of the evaluation experiment, the four experts generated 31 maps in total and selected 61 paths of interest from the maps. Table 3 shows the distribution of paths selected from the maps and evaluated by the subjects, classified on the basis of the general evaluation. According to the results, the number of paths classified in the higher two levels was 30 for A: Interesting and 22 for B: Ordinary but important, which totals 52 , occupying $85 \%$ of all the paths evaluated. Thus, we can conclude that it is possible with the tool to generate maps or paths sufficiently meaningful for experts. The extremely small number of D: Obviously wrong is attributable to the removal of unnecessary paths before the evaluation. However, this is conceivably not so problematic since the ratio of the unnecessary paths was about $70 \%$ to $80 \%$ of the paths existing just after map generation, and the paths can be removed by a simple operation $^{2}$. While this result may seem to be rough and subjective, note here that our research goal is not formal analysis of ontologies from the point of views of ontology engineers and computer scientists but supporting domain experts to understand contents of ontologies. Different from formal analysis, content understanding is

\footnotetext{
${ }^{1}$ The numbers of sub concepts of Environmental destruction problems and Tradeoff problems are 43 and 16 respectively. And the subjects can chose two kinds of focal points and two kinds of exploration methods.

${ }^{2}$ For example, when a node close to the center of a map is selected and paths are removed, all subpaths of the paths are removed, so that about $10 \%$ to $50 \%$ of all the paths are removed.
} 
essentially subjective task. Thus, the evaluation method cannot avoid being subjective to some extent. Although subjective evaluations would be understood to be ad hoc and incomplete, the very fact that domain experts evaluate it as good/useful, which is subjective, is meaningful to them, and hence to our research. Therefore, we believe our evaluation result should not be taken as meaningless but taken as demonstrating some positive evaluation that suggests the benefit of our approach.

Then, using the maps generated by reproducing the originating scenarios for ontology enrichment by the detailed exploration described in Section 4.2.2 as "anticipated maps," we quantitatively compared the anticipated maps with the maps generated by the subjects (after removing unnecessary paths) in the experiment to evaluate whether maps having meaningful contents other than the scenarios anticipated at the time of ontology construction were generated through the experiment. We compared them through some calculations using the numbers of concepts and relationships which are included in paths of the anticipated maps, the maps generated and selected by subjects and both of them. They are shown as N, M and $\mathrm{N} \cap \mathrm{M}$ respectively in Fig.7. The results of the evaluation were as follows. First, of the paths included in the anticipated maps (it is calculated by " $(\mathrm{N} \cap \mathrm{M}) / \mathrm{N}$ " in Fig.7), about $50 \%$ of them were included in the maps generated by the experts. This ratio indicates the ratio of problem structures that matched the paths of interests of experts in different domains among the problem structures anticipated as typical scenarios. Although the task settings in this experiment were not intended to reproduce the anticipated maps, conceivably, overlapping interests in the wide domain of biofuels appeared as overlapping interests among experts in different domains. On the other hand, the ratio of paths not included in the anticipated maps among the paths generated and selected by the experts, i.e., the ratio of paths not anticipated from the typical scenarios (it is calculated by " $(\mathrm{M}-\mathrm{N} \cap \mathrm{M}) / \mathrm{M}$ " in Fig.7), was about $75 \%$. We think the result is enough to show that three quarters are new paths and the other quarter is included in the anticipated paths. Although it is difficult to objectively claim what is the best rate, it is meaningful enough to claim a positive support for the developed tool. This suggests that the tool has a sufficient possibility of presenting unexpected contents and stimulating conception by the user. In other words, this suggests that, by organizing the contents of the scenarios as generalized concepts in the ontology instead of directly storing the contents in a computer, more meaningful conceptual maps were generated compared with the case where the contents were stored intentionally in a computer.

Furthermore, the experts who served as subjects left positive comments about the maps they generated, such as: "Biomass resource production obviously involves human labor. However, we tend to focus on the material flow and environmental load control (restriction of greenhouse gas emissions, prevention of water pollution, and suppression of soil degradation) and tend to forget about the presence of human beings who support it." or "Normally, I wouldn't have noticed the path to sea pollution," indicating the possibility that the tool can contribute to overviewing problems or stimulating conception assistance. Also, in discussions after the experiment, we received one opinion: "It is useful that relationships in various domains are expressed in maps based on a single ontology." That is, we confirmed 
that the ontology exploration could serve to clarify relationships of knowledge, which tended to be segmented, and contribute to assisting in an interdisciplinary integrated understanding of the entire field.

\section{Related Works}

The novelty of our study lies in neither ontology visualization nor ontology navigation. The feature of our approach is an ontology exploration according to the users' viewpoints, for supporting domain experts to understand ontologies. We do not suppose to argue for novelty of the techniques which are used for visualization of the result of ontology exploration. While many researchers discuss techniques for ontology exploration and visualization since the early 2000s [7], they focus on formal aspects of ontology from the point of views of ontology engineers. Our idea is application of the techniques to bridge gaps between ontology and domain experts. Originally, our ontology editor (Hozo) have had sophisticated user interfaces for ontology visualization and navigation. It also got favorable comments such as from users in a workshop for comparing several ontology development tools. [8] mentions the comment as "it's not surprising that users liked the visual aids that some of the tools provided, such as Hozo's interactive graphs". However, in one of our research projects, such an interface has been proven to be insufficient for domain experts of sustainability science to understand the content of the ontology which is one of the significant goals. In other words, all the existing visualization tools assuming to allow users to see the entire structure of the ontology are not appropriate for domain experts to understand ontology. We therefore investigated the ontology exploration tool through this experience, and then it was well received by the domain experts. While it might be possible to generate paths using existing ontology navigation tools by choosing ways to trace in details, we suppose it is difficult to stimulate domain expert's way of thinking through divergently explorations because most of them are not designed for domain experts. While our tool uses concept maps as a format for visualization because it is familiar to domain experts, we have no intention to claim a novelty of the visualization method. The purpose of our tool is not concept map exploration but ontology exploration for understanding the ontology. A feature of our tool is that concept maps are generated from an ontology which systematizes domain knowledge as general as possible. It enables the users generate maps which includes contents anticipated by the developer of ontology.

Some researchers have developed tools for ontology exploration. TGVizTab (TouchGraph Visualization Tab) also supports a visualization which is similar to our tool. It aims for enhancement of clarification, verification and analysis of an ontology [9]. On the other hand, the features of our tool are functions for ontology exploration such as control of range of visualization, highlighting of their intensions and so on according to their viewpoints because we focus not on visualization but on exploration according to the users' viewpoints. Bosca develops a Protégé plug-in, called Ontosphere 3D, which displays an ontology on a 3D space [10]. It allows the user to choose three kinds of visualization methods according his/her propose. The feature of this tool is a user definable set of ontology entities (concepts and relations) called Logical View. It is used for forming a hyper-surface in the multi-dimensional 
ontology space according to user's intensions. Although this approach to manage viewpoints is similar to our tool, it is different from the purpose of our tool that Ontosphere3D aims to provide ontology designers with a flexible instrument for effective representation and modeling of an ontology. Noppens proposes rendering techniques which combines visual analytics with interactive exploration for large scale structured data on the web [11]. When users are exploring an ontology, this tool clusters related entities according to their class hierarchies and inheritance information and visualize the result as clusters. It supports to discover hidden connections between individuals. While this tool focuses on exploration for large ontology with its instances (individuals), our tool aims at exploration for an ontology without instances. Helim also proposes an approach for interactive discovery relationships via the Semantic Web [12]. Tane discusses query-based multicontext theory for browsing ontologies [13]. In his approach, views for navigation are defined by sets of queries to knowledge bases. Though the search path function of our tool shares the same idea with them, it uses only simple combinations of relationships in an ontology because we focus on understanding the ontology. Christopher et.al discusses a constructive exploration of an ontology using world view and perspective [15]. Their work focuses on creating tight domain hierarchies from Folksonomies such as Wikipedia using mining techniques with some viewpoints. Exploring the knowledge space in Wikipedia is discussed as one of the techniques for mining. On the other hand, our approach focuses on understanding content of an ontology according to view points of domain experts.

\section{Concluding Remarks and Future Work}

This paper proposed divergent exploration to bridge a gap between an ontology which systematizes knowledge in generalized formats and domain experts who tend to understand the knowledge from domain-specific viewpoint. And we developed an ontology exploration tool for supporting users to explore an ontology divergently according to their intensions and viewpoints. This tool was applied to knowledge structuring of sustainability science. Through the experience, the tool was well received by domain experts in the domain and got favorable comment that it could contribute to proper structuring of knowledge across multiple domains. Then, we evaluated the tool through an experiment in cooperation with domain experts who are unfamiliar with ontology. As the result, we could make sure that domain experts could obtain meaningful knowledge for themselves as conceptual chains through the divergent exploration of ontology using the tool. The conceptual chains generated in the experiment included about $75 \%$ paths which were not supposed when the ontology was constructed. That is, we can say that the tool stimulated their way of thinking and contributed to obtaining unexpected conceptual chains which they have never thought. It is considered that the result is caused our approach for knowledge systematization that we should describe knowledge not as simple instances but as generalized concepts and relationships in an ontology. Furthermore, a domain expert who joined the experiment proposed us that our tool could be applicable for consensus-building among stakeholders in a workshop. It suggests that the tool 
clearly represents differences of thinking among stakeholders as differences of their viewpoints and conceptual chains generated by each of them so that they could understand ways of thinking of each other.

Future plan includes improvement of our tool to support more advanced problems such as consensus-building, policy-making and so on. We believe divergent exploration from multiple viewpoints contributes to making clear differences among standpoint, way of thinking and so on. For this purpose, we suppose to consider more intuitive user interfaces and visualizations for comparison between viewpoints. We also plan to be apply the ontology exploration tool for ontology refinement. In practice, we could use the tool to confirm whether the Biofuel ontology captures scenarios which were given by a domain expert and found some error in the ontology. We suppose it could be used for ontology refinement tool by domain experts to find not syntactic errors but content level faults in an ontology. We are trying to adopt this approach in a medical domain [15]. We think an evaluation of our tool on other ontologies is also important. Especially a large and complex ontology in OWL is a main target of the evaluation. While the system supports exploration of OWL ontologies, it does not support complex axioms in OWL. We will soon publish a new version of ontology explanation tool using OWL API with a reasoner.

\section{Acknowledgements}

This work was supported by Grant-in-Aid for Young Scientists (A) 20680009, the Global Environment Research Fund (Hc-082) of the Ministry of the Environment, Japan.

\section{References}

1. Gruber, T.: A translation approach to portable ontology specifications. In: Proc. of JKAW 1992, pp. 89-108 (1992)

2. Smith, B., et al.: The OBO Foundry: coordinated evolution of ontologies to support biomedical data integration. Nature Biotechnology 25(11), 1251-1255 (2007)

3. Kozaki, K., et al.: Hozo: An Environment for Building/Using Ontologies Based on a Fundamental Consideration of "Role" and "Relationship". In: Gómez-Pérez, A., Benjamins, V.R. (eds.) EKAW 2002. LNCS (LNAI), vol. 2473, pp. 213-218. Springer, Heidelberg (2002)

4. Mizoguchi, R., et al.: A Model of Roles within an Ontology Development Tool: Hozo. J. of Applied Ontology 2(2), 159-179 (2007)

5. Kozaki, K., et al.: Role Representation Model Using OWL and SWRL. In: Proc. of 2nd Workshop on Roles and Relationships in Object Oriented Programming, Multiagent Systems, and Ontologies, Berlin, July 30-31 (2007)

6. Kumazawa, T., et al.: Toward Knowledge Structuring of Sustainability Science Based on Ontology Engineering. Sustainability Science 4(1), 99-116 (2009)

7. Katifori, A., Halatsis, C., et al.: Ontology visualization methods - a survey. ACM Computing Surveys (CSUR) 39(4) (2007)

8. Noy, N.F., et al.: The CKC Challenge: Exploring Tools for Collaborative Knowledge Construction. IEEE Intelligent Systems 23(1), 64-68 (2008) 
9. Alani, H.: TGVizTab: An ontology visualization extension for Protégé. In: Proc. of Knowledge Capture (K-Cap 2003), Workshop on Visualization Information in Knowledge Engineering, Sanibel Island, Florida (2003)

10. Bosca, A., Bonino, D.: Ontology Exploration through Logical Views in Protégé. In: Wagner, R., Revell, N., Pernul, G. (eds.) DEXA 2007. LNCS, vol. 4653, pp. 465-469. Springer, Heidelberg (2007)

11. Noppens, O., Liebig, T.: Understanding Interlinked Data - Visualising, Exploring, and Analysing Ontologies. In: Proc. of International Conferences on Knowledge Management and New Media Technology (I-KNOW 2008), Graz, Austria, pp. 341-348 (2008)

12. Heim, P., Lohmann, S., Stegemann, T.: Interactive Relationship Discovery via the Semantic Web. In: Aroyo, L., Antoniou, G., Hyvönen, E., ten Teije, A., Stuckenschmidt, H., Cabral, L., Tudorache, T. (eds.) ESWC 2010. LNCS, vol. 6088, pp. 303-317. Springer, Heidelberg (2010)

13. Thomas, C., et al.: Growing Fields of Interest - Using an Expand and Reduce Strategy for Domain Model Extraction. In: IEEE/WIC/ACM International Conference on Web Intelligence and Intelligent Agent Technology 2008, vol. 1, pp. 496-502 (2008)

14. Tane, J., Cimiano, P., Hitzler, P.: Query-based multicontexts for knowledge base browsing: An evaluation. In: Schärfe, H., Hitzler, P., Øhrstrøm, P. (eds.) ICCS 2006. LNCS (LNAI), vol. 4068, pp. 413-426. Springer, Heidelberg (2006)

15. Ohta, M., Kozaki, K., Mizoguchi, R.: A quality assurance framework for ontology construction and refinement. In: Mugellini, E., Szczepaniak, P.S., Pettenati, M.C., Sokhn, M. (eds.) AWIC 2011. Advances in Intelligent and Soft Computing, vol. 86, pp. 207-216. Springer, Heidelberg (2011) 